\title{
Efecto de la hiperglucemia en el síndrome coronario agudo y sus implicaciones en el tratamiento antiagregante plaquetario
}

\author{
Juan Pablo Pérez-Bedoya' ${ }^{1}$ Natalia Gallego-Lopera', Cristian Arbey Velarde-Hoyos's ${ }^{3}$ Liliana Franco-Hincapié4, Kelly \\ Johanna Betancur-Salazar ${ }^{5}$, Ana Victoria Valencia-Duarte ${ }^{6}$
}

\section{RESUMEN}

La enfermedad cardiovascular representa, según los datos de la Organización Mundial de la Salud, la principal causa de muerte asociada con factores de riesgo como el tabaquismo, el sedentarismo, la hipertensión, la dislipidemia y la diabetes mellitus. Precisamente, esta última enfermedad es una de las que más se relaciona con la aparición, la progresión y las complicaciones de un evento coronario. La hiperolucemia potencia diferentes vías bioquímicas y celulares como la del sorbitol, el factor nuclear $\mathrm{k} \beta$, la formación de productos finales de glicación avanzada, la vía de la proteína cinasa C y el estrés oxidativo, que terminan favoreciendo en el paciente coronario un estado proinflamatorio y procoagulante, que se asocia con un peor pronóstico y agrava la lesión miocárdica; además, inhibe y compite con la acción de Ios antiagregantes plaquetarios, generando resistencia no solo a estos sino también a la terapia trombolítica. Por lo anterior, se hace necesario generar una actualización del tema, para sensibilizar a la comunidad médica sobre la importancia del control glucémico, sobre todo en pacientes con cardiopatía isquémica, y así mejorar las estrategias de control. Se realizó la búsqueda bibliográfica en PubMed, de una forma estructurada, no sistemática. Se incluyeron artículos publicados en inglés y español, sin restricción por fecha de publicación.

\section{PALABRAS CLAVE}

Agregación Plaquetaria; Diabetes Mellitus; Hiperolucemia; Síndrome Coronario Agudo

1 Facultad de Ciencias de la Salud. Institución Universitaria Colegio Mayor de Antioquia, Colombia

PhD(c) en ciencias médicas. Universidad Pontificia Bolivariana, Grupo Biología de Sistemas, Escuela de Ciencias de la Salud, Facultad de Medicina, Medellín, Colombia.

Magíster en Bioingeniería. Universidad Pontificia Bolivariana, Grupo Biología de Sistemas, Escuela de Ciencias de la Salud, Facultad de Medicina, Medellín, Colombia.

PhD en Epidemiología. Docente Universidad Pontificia Bolivariana, Grupo Biología de Sistemas, Escuela de Ciencias de la Salud, Facultad de Medicina, Medellín, Colombia.

Residente de Medicina Interna, Universidad Pontificia Bolivariana, Colombia.

PhD Biología. Docente Universidad Pontificia Bolivariana, Grupo Biología de Sistemas, Escuela de Ciencias de la Salud, Facultad de Medicina, Medellín, Colombia.

Correspondencia: Ana Victoria Valencia Duarte; anavictoria.valencia@upb.edu.co

Recibido: mayo 11 de 2018

Aceptado: agosto 13 de 2018

Cómo citar: Pérez-Bedoya JP, Gallego-Lopera N, Velarde-Hoyos CA, Franco-Hincapié L, Betancur-Salazar KJ, Valencia-Duarte AV. Efecto de la hiperglucemia en el síndrome coronario agudo y sus implicaciones en el tratamiento antiagregante plaquetario. latreia. 2019 Abr-Jun; 32(2):113-125. D0I 10.17533/udea.iatreia.04. 


\section{SUMMARY}

Effect of hyperglycemia on acute coronary syndrome and the implications of antiplatelet therapy

According to data from the World Health Organization, cardiovascular disease is the main cause of death associated with risk factors such as smoking, sedentary lifestyle, hypertension, dyslipidemia and diabetes mellitus. Precisely, this last disease is one of the most related to the appearance, progression, and complications of a coronary event. Hyperglycemia potentiates different biochemical and cellular pathways such as sorbitol, the nuclear factor $k \beta$, the formation of advanced glycation end products, the protein kinase $C$ pathway and oxidative stress, which end up favoring in the coronary patient a proinflammatory state and procoagulant, which is associated with a worse prognosis and agoravates myocardial injury; in addition, it inhibits and competes with the action of platelet antiaggregants, generating resistance not only to these but also to thrombolytic therapy. Therefore, it is necessary to generate an update of the topic, to sensitize the medical community about the importance of glycemic control, especially in patients with ischemic heart disease and thus improve control strategies. The bibliographic search was carried out in PubMed, in a structured, non-systematic way. Articles published in English and Spanish were included, without restriction by publication date.

\section{KEY WORDS}

Acute Coronary Syndrome; Diabetes Mellitus; Hyperglycemia; Platelet Aggregation

\section{INTRODUCCIÓN}

La enfermedad cardiovascular (ECV), y específicamente la cardiopatía coronaria, es la principal causa de muerte en el mundo de acuerdo con la Organización Mundial de la Salud (OMS), estas ocasionaron 17,5 millones de muertes en el 2012 (1). El diagnóstico de diabetes mellitus (DM) se considera como uno de los principales factores de riesgo para el desarrollo de la cardiopatía isquémica (2), siendo
Ia enfermedad coronaria de etiología aterosclerótica la principal causa de muerte en pacientes diabéticos (3).

El tratamiento antiagregante plaquetario con ácido acetilsalicílico y clopidogrel es la terapia principal utilizada en pacientes con síndrome coronario agudo (SCA), con el objetivo de reducir eventos cardiovasculares adversos (4-6). Sin embargo, existen evidencias sobre el fracaso de esta terapia dual, explicada por el mayor riesgo de reincidencia en eventos cardiovasculares (7), con reportes de resistencia que van desde el 5 al $45 \%$ en la población (4). Razón por la cual en el mundo, incluido Colombia, esta resistencia constituye un gran impacto en el estado de salud de los pacientes, ocasionando mayores complicaciones, un peor pronóstico y un aumento en el costo de la atención en salud (4).

Un factor clínico común, que predispone a la resistencia a los antiagregantes plaquetarios y hace más precoz la cardiopatía coronaria, es la hiperolucemia. Esta propicia la activación de rutas bioquímicas que generan un ambiente proinflamatorio y procoagulante en el paciente, como la generación de especies reactivas de oxígeno, la activación de proteína cinasa C (PKC), formación de productos finales de glicación avanzada (PGA), la activación del factor nuclear $\kappa$ B, la vía del sorbitol y la vía de las hexosaminas $(5,8)$. Esta revisión pretende hacer un compendio de la información encontrada en la literatura médica, con respecto a la relación existente entre la hiperglucemia y su efecto sobre el SCA, con el fin de poder establecer estrategias de promoción de la salud y prevención de la enfermedad.

\section{METODOLOGÍA}

Se realizó una revisión narrativa definiendo unos subtemas para el desarrollo del contenido. La búsqueda se hizo en PubMed con las palabras clave: hyperolycemia, acute coronary syndrome, platelet aogreogation y diabetes mellitus. Se incluyeron los artículos que abordaran los temas propuestos, publicados tanto en inglés como en español y que estuvieran disponibles en texto completo. No se hizo restricción por año de publicación. Dado el tipo de revisión no se contabilizó la cantidad de artículos recuperados. 


\section{EPIDEMIOLOGÍA DE LA ENFERMEDAD CARDIOVASCULAR}

Las enfermedades cardiovasculares hacen parte del grupo de enfermedades crónicas no trasmisibles, donde la más frecuente es la cardiopatía isquémica, la cual causa un número mayor de muertes que cualquier otro trastorno $(1,9,10)$. Además, es una de las más importantes causas de incapacidad debido al fenómeno de envejecimiento poblacional con cambios en el estilo de vida, como la alimentación con alto contenido de grasas y carbohidratos, el aumento del estrés y la urbanización de las medianas y grandes ciudades (11). Todo lo anterior conlleva a un aumento del coste económico para el sistema de salud (12), haciendo que la enfermedad coronaria represente un importante problema de salud pública en el mundo (9).

De acuerdo con la OMS, en el año 2012 se reportaron 17,5 millones de muertes por ECV (13), lo que representó el $31 \%$ de las muertes registradas en el mundo. De estas, 7,4 millones se debieron a la cardiopatía coronaria, con un mayor número de casos en países de ingresos medios y bajos $(1,14)$. En América la cardiopatía isquémica causó aproximadamente 82,9 muertes por cada 100.000 habitantes el año 2010, donde América Latina aportó 57,3 muertes por cada 100.000 habitantes (15).

Para las diversas poblaciones en el ámbito mundial, los factores de riesgo convencionales para la ECV son el tabaquismo, el sedentarismo, la alimentación no saludable, el consumo excesivo de alcohol, la hipertensión arterial, hiperlipidemia, sobrepeso u obesidad, hiperglucemia y DM (9). La mayoría de estas comorbilidades conducen a un estado inflamatorio crónico que favorece el proceso aterotrombótico (10). También influyen factores ambientales, genéticos, epigenéticos y clínicos, que en últimas son los desencadenantes de la aparición, progresión y mal pronóstico de la enfermedad coronaria $(1,16)$.

La enfermedad coronaria se correlaciona con la presencia de alteraciones en el metabolismo de la glucosa, siendo la cardiopatía isquémica de etiología aterosclerótica la principal causa de muerte en pacientes diabéticos. La DM está presente en aproximadamente un tercio de los pacientes diagnosticados con SCA (3), y según la Federación Internacional de Diabetes, esta afecta actualmente a 425 millones de personas en el mundo y se estima que para el año 2045 serán aproximadamente 629 millones de personas (17).

\section{DEFINICIÓN DEL SÍNDROME CORONARIO AGUDO}

La cardiopatía isquémica se caracteriza por un suministro insuficiente de oxígeno $y$ un desequilibrio entre el aporte y la demanda por parte de las arterias coronarias. La causa más común es la enfermedad ateroesclerótica (18), que dependiendo de la oclusión del flujo sanguíneo puede tener diferentes presentaciones clínicas, que van desde una angina estable a un SCA (19). Esta última presentación a su vez, se clasifica en angina inestable o infarto agudo de miocardio (IAM) con o sin elevación del segmento ST $(9,18,20)$.

Para definir un IAM se establecen criterios que incluyen la elevación en la sangre de biomarcadores cardiacos, acompañados de cambios electrocardiográficos y signos y síntomas característicos de isquemia miocárdica, como el dolor torácico $(18,21)$. En el electrocardiograma se puede distinguir el IAM con $y$ sin elevación del segmento ST. En el primero, se encuentra una oclusión completa de la arteria coronaria y requiere un tratamiento urgente enfocado en recuperar el flujo sanguíneo, con procedimientos farmacológicos y mecánicos invasivos. En el segundo caso, la arteria coronaria no está ocluida completamente, por lo cual el tratamiento se basa en antiagregantes plaquetarios $y$ anticoagulantes $(12,21)$.

\section{LA HIPERGLUCEMIA Y SU RELACIÓN CON EL SÍNDROME CORONARIO AGUDO}

La hiperglucemia aguda y crónica, con o sin previo diagnóstico de DM, se asocian al aumento del riesgo de eventos adversos, a una mayor estancia hospitalaria e incluso a una mayor probabilidad de muerte en los pacientes con SCA $(22,23)$. Así la hiperglucemia predice un peor pronóstico y agrava la lesión miocárdica (24), lo que afecta la recuperación de los pacientes y genera complicaciones a mediano y laroo plazo (25). Además, las concentraciones anormales de hemoglobina glicada ( $\mathrm{HbA} 1 \mathrm{C}$ ) se asocian con un mayor riesgo de eventos cardiovasculares (26). 
Se estima que dos de cada tres muertes en la población diabética es debida a ECV, de la cual aproximadamente un $40 \%$ corresponde a la enfermedad coronaria isquémica, $15 \%$ a cardiomiopatías, principalmente insuficiencia cardiaca y $10 \%$ a isquemia cerebral. Este riesgo elevado de desarrollar ECV se debe principalmente a la disfunción endotelial generada dentro de las alteraciones metabólicas propias de la diabetes (27). Además, se ha estimado que por cada incremento en $1 \%$ de la $\mathrm{HbA} 1 \mathrm{C}$ se incrementa el riesgo cardiovascular entre un 11-16\%, y que a partir de una glucemia en ayunas de $105 \mathrm{mg} / \mathrm{dl}$, por cada 18 $\mathrm{mg} / \mathrm{dl}$ que esta aumente se eleva el riesgo de padecer ECV aproximadamente en un $12 \%$ (27).

Ha existido controversia acerca de si la prediabetes per se incrementa el riesgo cardiovascular en los que la padecen o si, por el contrario, este riesgo es potenciado por la coexistencia de la hipertensión arterial. En un estudio realizado por $\mathrm{Li}$, et al., en donde se incluyeron 7.121 pacientes, se encontró que no existen diferencias estadísticamente sionnificativas $(p>0,05)$ respecto al número de eventos y la gravedad de la enfermedad coronaria entre pacientes prediabéticos y normoglucémicos. Sin embargo, al evaluar y comparar la gravedad coronaria y el pronóstico clínico en los pacientes prediabéticos e hipertensos respecto a normoglucémicos y normotensos, se obtuvo una diferencia estadísticamente significativa (28).

La hiperglucemia por estrés se define como la concentración plasmática de glucosa en ayunas mayor o igual a $126 \mathrm{mg} / \mathrm{dL}$, o determinada en cualquier momento mayor o igual $140 \mathrm{mg} / \mathrm{dL}$ (200 mg/dL para otros autores) (29), que se encuentra en pacientes hospitalizados o críticamente enfermos, sin evidencia previa de diabetes $(23,30)$. Esta condición se explica por el incremento de hormonas contrarreguladoras circulantes (cortisol, glucagón, hormona del crecimiento $y$ catecolaminas) $y$ de citoquinas proinflamatorias que interfieren con el metabolismo de los carbohidratos, lo que lleva a una excesiva producción hepática de glucosa y a una recaptación disminuida de ella en los tejidos periféricos. Por otro lado, situaciones como el uso de glucocorticoides, la sepsis, el trauma y los procesos inflamatorios en general, inhiben la secreción, liberación y acción de la insulina (22), aumentando las concentraciones plasmáticas de glucosa durante la estancia hospitalaria.
En estos casos la determinación de hemoglobina glicada es clave para diferenciar pacientes con verdadera hiperolucemia por estrés, de aquellos diabéticos no diagnosticados (22).

Las alteraciones del metabolismo de la glucosa son muy comunes en pacientes diagnosticados con SCA. En un estudio realizado en Bulgaria en el año 2011, se encontró que el $78 \%$ de los pacientes con enfermedad arterial coronaria presentaban algún tipo de disglucemia (24). Adicionalmente, se ha reportado que los pacientes con SCA presentan hiperglucemia de estrés con una frecuencia entre el 25 y 50 \% (31). Estudios epidemiológicos han hallado que el riesgo relativo de muerte intrahospitalaria en pacientes con SCA y DM o, hiperglucemia, fue 3,9 veces mayor que el de los normoglucémicos $(25,32)$.

El papel que desempeña la hiperglucemia en la aparición y progresión de las complicaciones en los pacientes coronarios es muy diverso, e incluye mecanismos moleculares, bioquímicos y celulares. Entre estos, se encuentra la formación y acción de los productos finales de glicación avanzada (PGA) sobre sus receptores, autooxidación de la glucosa, incremento de la vía del sorbitol, activación de la PKC, aumento del estrés oxidativo, activación del factor nuclear kB y la producción de isoprostanos, eventos que en conjunto llevan a la formación de un ambiente proinflamatorio, procoagulante $y$ vasoconstrictivo, lo que favorece la aparición de las complicaciones del SCA $(3,5,8,33)$. Estos efectos dependen no solo de la concentración de glucosa, sino del periodo de exposición a la hiperglucemia (3), permitiendo la activación de estas diferentes rutas bioquímicas que favorecen la progresión y evolución de la cardiopatía en el paciente (Figura 1).

En el caso de la hiperglucemia aguda, sus efectos deletéreos están dados en su gran mayoría por la activación del sistema simpático y de las principales rutas metabólicas que llevan a estados proinflamatorios y procoagulantes; en la hiperglucemia crónica, además de lo anterior, se generan PGA con aumento de síntesis de tromboxano A2, lo que potencia, aún más, el estado procoagulante (34).

A continuación, se describen los principales mecanismos activados en los estados de hiperglucemia y su relación con el SCA. 


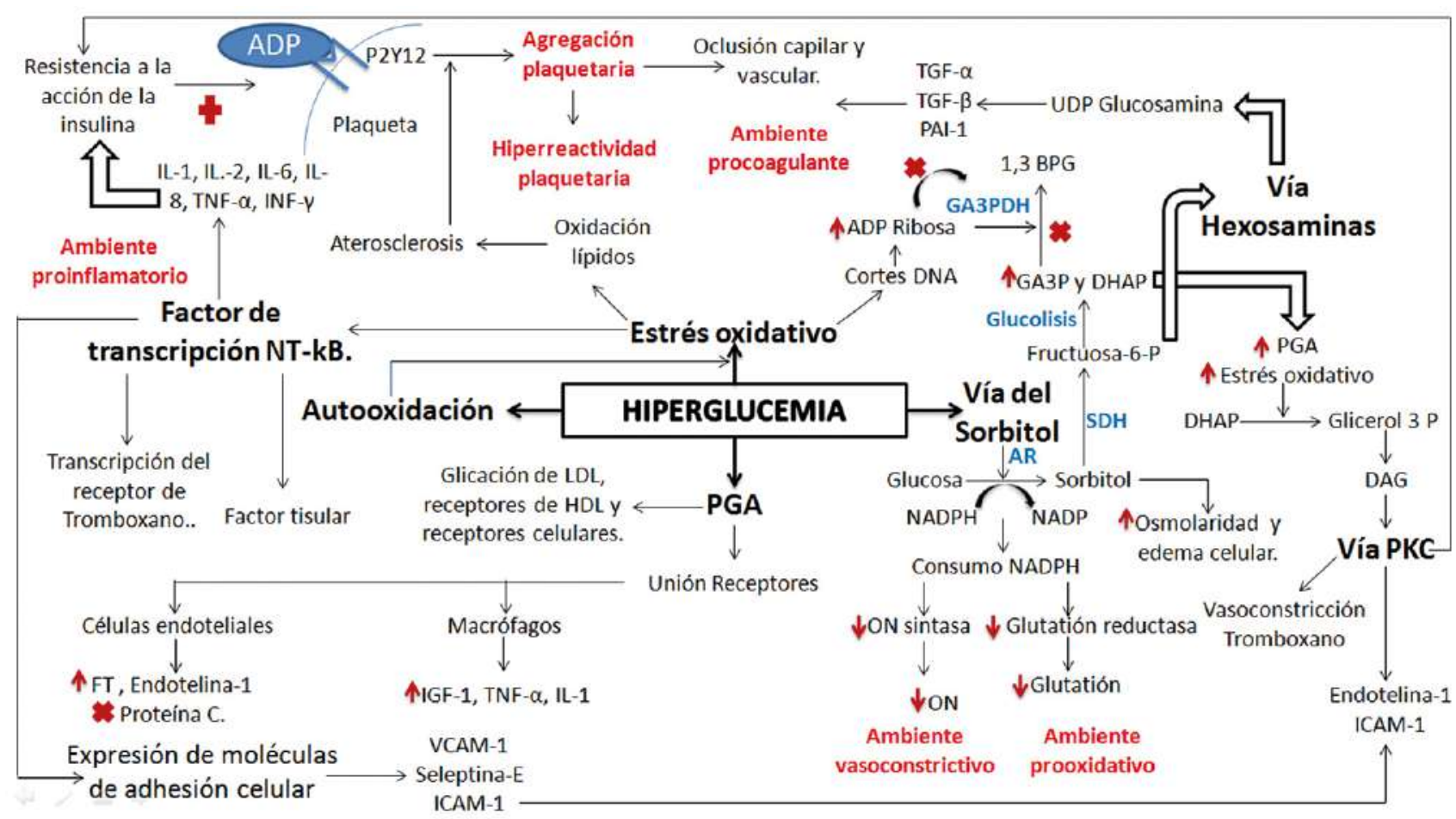

Figura 1. Principales mecanismos fisiopatológicos implicados en la generación del estado protrombótico, procoagulante, prooxidativo y proinflamatorio a partir de la hiperglucemia y su relación con el evento coronario

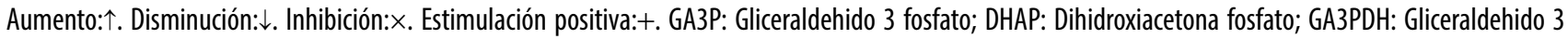
fosfato deshidrogenasa; AR: Aldosa reductasa; SDH: Sorbitol deshidrogenasa; PGA: Productos de glicación avanzada; PKC: Proteína cinasa C; PAl-1: Inhibidor del activador tisular del plasminógeno; TNF-a: Factor de necrosis tumoral alfa; INF-y: Interferon gamma; IGF: Factor de crecimiento insulínico; TGF: Factor de crecimiento transformante; ON: Óxido nítrico; DAG: Diacilglicerol; /CAM-1: Molécula de adhesión intercelular-1;VCAM-1: Molécula de citoadhesión vascular-1; 1.3 BPG: 1,3 Bifosfoglicerato; NADP: Nicotinamida adenina dinucleótido fosfato-forma oxidada; NADPH: Nicotinamida adenina dinucleótido fosfato-forma reducida; ADP: Adenosín difosfato. Fuente: elaboración Propia

\section{Estrés Oxidativo}

Las altas concentraciones de glucosa sanguínea favorecen la producción de radicales libres de oxígeno, principalmente radical superóxido $\left(\mathrm{O}_{2}^{-}\right)(35)$, los cuales se generan cuando el oxígeno se reduce de manera incompleta durante la fosforilación oxidativa a nivel mitocondrial $(35,36)$, lo cual produce daño celular por estrés oxidativo debido a un desbalance entre radicales libres y los antioxidantes (37). Esta condición genera oxidación de compuestos celulares como proteínas y lípidos, lo que favorece aún más el proceso aterosclerótico. Además genera quiebres en el DNA incrementando la expresión de ADP ribosa que bloquea la enzima gliceraldehído 3 fosfato deshidrogenasa (GA3PDH) y aumenta el gliceraldehído 3 fosfato (GA3P) (35), y se activan así otras rutas bioquímicas como la vía del sorbitol, la de las hexosaminas y la $\operatorname{PKC}(35,37)$, que finalmente conducen al daño vascular.

\section{La vía del sorbitol}

Esta se activa cuando las concentraciones de glucosa aumenta en respuesta al bloqueo de la glucolisis por 
causa del estrés oxidativo, en consecuencia, la enzima aldosa reductasa (que presenta baja afinidad por la glucosa cuando sus concentraciones plasmáticas son normales) $(37,38)$, cataliza la conversión de glucosa a sorbitol, con el consumo de una molécula de NADPH (35-38) que en condiciones normales es indispensable para la generación de antioxidantes como el glutatión y la producción de óxido nítrico (35). Por lo anterior, se establece un ambiente prooxidante y vasoconstrictor.

EI sorbitol es poco permeable a la membrana celular, lo que incrementa la osmolaridad $y$ favorece el edema celular (35-38). La vía del sorbitol, además, genera fructosa 6 fosfato (F6P) que se acopla a la glucólisis, ocasionando una retroalimentación positiva al aumentar el GA3P, para comenzar nuevamente el ciclo y producir más estrés oxidativo y glicación de macromoléculas $(35,38)$, todo lo anterior favorece la aparición de las complicaciones del SCA.

\section{Vía de las hexosaminas y de la proteína cinasa $\mathrm{C}$}

EI incremento de la F6P activa una ruta metabólica poco usual, la vía de las hexosaminas, la cual da origen a UDP-glucosamina, molécula asociada con el aumento en la expresión de genes como los del factor de crecimiento transformante alfa (TGF- $\alpha$ ), factor de crecimiento transformante $\beta 1$ (TGF- $\beta 1$ ) y del inhibidor del activador del plasminógeno PAI-1 (38), lo que contribuye a generar oclusión capilar y vascular (35) en el paciente coronario.

Al mismo tiempo que aumenta la concentración de GA3P, se incrementa la de dihidroxiacetona fosfato (DHAP) (38), que luego es reducida a glicerol 3 fosfato $y$, junto con la acetil coA, forman ácidos grasos. Esto propicia la formación del diacilglicerol (DAG) y activa la vía de la PKC. Esta enzima es un importante mensajero en la transducción de señales intracelulares, que terminan con la expresión de diversos genes (38) como la fibronectina, el colágeno tipo IV, el PAI-1 y el TGF $\beta 1(35,38,39)$. De modo adicional, la activación de la PKC reduce la síntesis de óxido nítrico $y$ aumenta la de endotelina-1, potenciando el ambiente vasoconstrictor y trombótico (35).

\section{Vía de los productos finales de glicación avanzada (PGA)}

Dependiendo del estado hiperglucémico y de su cronicidad, se generan los PGA que se correlacionan con la memoria metabólica y con las alteraciones celulares (40). Los PGA se forman a partir de reacciones no enzimáticas entre el grupo amino libre de la lisina de una proteína y la glucosa $(40,41)$. Esta glicación ocasiona modificaciones en las propiedades físico-químicas y biológicas de las proteínas, las cuales son reconocidas por receptores específicos en los macrófagos que inducen la producción de factor de crecimiento insulínico tipo 1(IGF-1), factor de necrosis tumoral alfa (TNF- $\alpha$ ), interleuquina 1 (IL-1) y por células endoteliales que generan el factor tisular, endotelina-1 e inhiben la proteína C (41), lo que lleva a un estado procoagulante $y$ proinflamatorio en el paciente con cardiopatía isquémica. La formación de los PGA también favorecen la aterosclerosis al glicar las lipoproteínas de baja densidad (LDL), haciéndolas más aterogénicas y fáciles de fagocitar por los macrófagos que están en el interior de la placa ateromatosa. Por otro lado, la glicación de las lipoproteínas de alta densidad (HDL) afecta su reconocimiento de parte del receptor, promoviendo la evolución de la placa (40).

\section{Vía del factor de transcripción nuclear NF- $\kappa B$}

Otro proceso activado por los niveles elevados de glucemia es la vía del factor de transcripción nuclear NF- $\kappa$ B. Este se encuentra localizado de forma inactiva en el citoplasma, unido a proteínas inhibidoras. Se activa tanto por procesos de fosforilación, mediante señales provenientes de citoquinas proinflamatorias como IL- $1 \beta$ y TNF- $\alpha$, como por estrés oxidativo y aumento de calcio intracelular, entre otros $(42,43)$. Estos estímulos permiten la liberación de la subunidad inhibitoria (42), posibilitando la translocación del NF$\kappa \mathrm{B}$ al núcleo para promover la transcripción de genes como el del factor tisular, las moléculas de adhesión celular (molécula de citoadhesión vascular 1 (VCAM1), molécula de adhesión intercelular 1 (ICAM-1) y selectina-E), el gen del receptor del tromboxano y citoquinas proinflamatorias como IL-1; IL-2, IL-6, IL-8, TNF- $\alpha$ e INF $\gamma(42,44,45)$.

Lo expuesto anteriormente ilustra la manera cómo las diferentes rutas activadas por la hiperglucemia en el paciente con SCA favorecen las complicaciones, debido a que se establecen mecanismos inflamatorios y procoagulantes, que se asocian a una mayor estancia hospitalaria, reincidencia de eventos coronarios $y$ un mayor riesgo de muerte (46). 


\section{TRATAMIENTO DEL SÍNDROME CORONARIO AGUDO}

El tratamiento del SCA se fundamenta en reestablecer la circulación coronaria, ya sea por medios mecánicos (intervención coronaria percutánea (ICP), derivación coronaria) o farmacológicos (trombolisis), así como en impactar sobre las diversas vías fisiopatológicas implicadas en la génesis y progresión del SCA; la terapia dual antiagregante plaquetaria (ácido acetil salicílico sumado a un inhibidor del P2Y12, siendo el clopidogrel el más usado) es el pilar del tratamiento, tanto en la fase aguda como en la crónica (33, 47-49).

\section{HIPERGLUCEMIA Y RESISTENCIA A LA TERAPIA ANTIAGREGANTE PLAQUETARIA}

La frecuencia de la resistencia a los antiagregantes plaquetarios en pacientes con SCA que han requerido ICP se encuentra entre el 5 y el $45 \%$ para el ácido acetilsalicílico y entre el 4 y $30 \%$ para el clopidogrel (4), en pacientes de diferentes poblaciones. Se presenta en ellos un mayor riesgo de recurrencia de eventos cardiovasculares como trombosis del stent, infarto o muerte súbita (50).

La resistencia a los antiagregantes plaquetarios puede determinarse a nivel bioquímico a través de la prueba por transmisión de luz (ATL), o a nivel clínico cuando los pacientes presentan recurrencia de un evento coronario (48). Esta resistencia puede ser explicada por factores genéticos, epigenéticos y clínicos como la falta de adherencia al tratamiento, dosificación inapropiada, interacciones farmacológicas, comorbilidades asociadas (33), variantes en genes que codifican para las proteínas involucradas en la farmacocinética y farmacodinamia de los antiagregantes (48, 51-57), entre otras.

Aunque esta terapia farmacológica ha demostrado su efectividad en el mejoramiento clínico de pacientes con SCA sometidos a ICP, en los pacientes diabéticos tratados con estos agentes antiplaquetarios se ha evidenciado una mayor frecuencia de eventos isquémicos recurrentes en comparación con pacientes con SCA sin DM (5). Angiolillo, et al. (2005), analizaron los valores de agregación plaquetaria en 60 pacientes diabéticos versus 60 no diabéticos con SCA en terapia clopidogrel/ácido acetilsalicílico, evidenciaron un aumento significativo de la agregación plaquetaria comparado con los pacientes no diabéticos ( $\mathrm{p}=$ 0,03 ), y en otro grupo, se observó un número mayor de no respondedores al clopidogrel en las 24 horas posteriores al inicio de la terapia en pacientes diabéticos $(\mathrm{p}<0,04)(58)$.

EI método más usado por los laboratorios clínicos y de investigación para evaluar la inhibición de la agregación plaquetaria en respuesta a los antiagregantes, es la ATL; específicamente, para determinar la resistencia al clopidogrel y al ácido acetil salicílico se utilizan los agonistas adenosín difosfato (ADP) y ácido araquidónico (AA), respectivamente. Se considera un paciente resistente al ácido acetil silícico cuando se obtiene una agregación mayor o igual al $20 \%$, y para el clopidogrel con una agregación mayor o igual al 50 o $70 \%(59,60)$.

Es importante resaltar que, si bien la ATL es considerada actualmente el estándar de oro, este no es un método estandarizado, pues presenta reproducibilidad limitada y complejidad en la preparación de la muestra, no cuenta con intervalos de referencia establecidos, programas de control interno y externo de calidad, $y$ tiene amplias fuentes de error en la fase preanalítica y analítica, entre otros (60).

\section{Papel de la hiperglucemia en la resistencia a los antiagregantes plaquetarios}

Otro mecanismo que potencia la resistencia a los antiagregantes plaquetarios es la hiperglucemia, $y$ por ende, la diabetes mellitus (33). Las altas concentraciones de glucosa plasmática desencadenan rutas bioquímicas que disminuyen la efectividad de estos fármacos, como disfunción endotelial, anormalidades en la coagulación y fibrinólisis, generación de trombina y la hiperreactividad plaquetaria (una de las más importantes), conduciendo a un aumento del riesgo de aterotrombosis (61-64).

\section{Hiperglucemia en la disfunción endotelial}

La disfunción endotelial se define como la incapacidad del endotelio para mantener una homeostasis vascular (63), generada por un desequilibrio entre la biodisponibilidad de óxido nítrico debido al estrés oxidativo y el aumento de la vasoconstricción que es debido, principalmente, a la endotelina-1 que es generada a partir de 
la activación de la PKC (65) y por la unión de los PGA a los macrófagos. Ante la presencia de hiperolucemia, las células endoteliales aumentan la expresión de moléculas de adhesión vascular como ICAM-1 y VCAM-1, y la secreción de citoquinas proinflamatorias, moléculas quimioatrayentes $y$ factores implicados en la trombosis, como PAI-1, tromboxano y factor tisular (63). La activación de la PKC en el endotelio vascular favorece la inflamación, angiogénesis, crecimiento celular y apoptosis, lo que conduce a la progresión de la lesión aterosclerótica y la disfunción endotelial, generando un ambiente protrombótico (65).

\section{Hiperglucemia y su efecto en la terapia trombolítica}

Dentro de las alteraciones relacionadas con la coagulación y la fibrinólisis en los pacientes hiperglucémicos, se encuentra un aumento del factor tisular debido a la presencia de procesos inflamatorios (65) y a la activación del factor de transcripción Nf-kB. Lo anterior favorece un aumento de la producción de trombina. Se generan además, otros fenómenos que evitan la degradación del coágulo (incremento en síntesis de fibrinógeno (63), una disminución en la biodisponibilidad del activador tisular del plasminógeno (t-PA) $(63,65)$ y un aumento del inhibidor del activador tisular del plasminógeno). En un estudio realizado en el año 2005, Dunn, et al.,demostraron que a una mayor concentración de $\mathrm{Hb} 1 \mathrm{Ac}$ son mayores los cambios estructurales en la conformación del coágulo, entre los que se observaron mayor densidad y menor porosidad. Se evidenció que la diabetes mellitus estaba involucrada tanto con el fracaso a la terapia antiagregante como a la terapia trombolítica en los pacientes con IAM (65).

\section{Hiperglucemia e hiperreactividad plaquetaria}

La hiperglucemia también favorece la hiperreactividad plaquetaria, puesto que altera la homeostasis del calcio por acción de las especies reactivas del oxígeno que estimulan la secreción de factores proagregantes $(64,65)$. Adicionalmente, el estrés oxidativo generado por la hiperoglucemia potencia la vía de señalización p38 MAPK, que activa la fosfolipasa citosólica $A_{2}$ y permite la formación de ácido araquidónico y la biosíntesis de tromboxano $\mathrm{A}_{2}$. Asimismo, la agregación inducida por el colágeno también es estimulada por la producción de especies reactivas de oxígeno (64). Por lo anterior, la hiperglucemia se puede considerar como un riesgo bioquímico al disminuir el efecto de los antiagregantes plaquetarios y promover la activación de las vías de señalización implicadas en empeorar el estado protrombótico del paciente con SCA.

Es importante aclarar que no solo la hiperglucemia está involucrada en cada uno de estos mecanismos fisiopatológicos; también se ha demostrado que la hipercolesterolemia incrementa la actividad plaquetaria, incluso más que la hipertrigliceridemia, debido a que el LDL oxidado sirve como ligando para el CD34 al promover la activación plaquetaria, formación de micropartículas de factor tisular derivadas de monocitos y el aumento de la megacariopoyesis, entre otros mecanismos (66).

Park, et al., encontraron que de un total de 1045 pacientes diabéticos medicados con ácido acetilsalicílico, 102 presentaron resistencia asociada a concentraciones de colesterol total $(p=0,013)$, LDL $(p=0,028) y$ colesterol no HDL $(p=0,008)$. En el análisis de regresión logística multivariado, solo el colesterol no HDL se asoció con la resistencia al medicamento en pacientes con DM y obesos (67). En otro estudio publicado por Grzegorz, et al., donde se incluyó a 52 pacientes con IAM con elevación del ST, se demostró que un nivel sérico más alto de lipoproteína A-I en fase aguda de IAM es un factor de riesgo independiente para la reactividad plaquetaria alta durante el tratamiento $(p=0,049)$; este hallazgo es novedoso y le atribuye una actividad distinta a la subfracción del HDL-C (68).

\section{MANEJO DE LA HIPERGLUCEMIA CRÓNICA Y SU IMPACTO EN LA ENFERMEDAD CORONARIA}

Se ha observado que el manejo intensivo del control glucémico reduce el riesgo de eventos microvasculares relacionados con la diabetes mellitus. En un estudio realizado por Holman, et al., se demostró que, además de reducir las complicaciones microvasculares, se lograba una reducción significativa del riesgo emergente de infarto de miocardio $(p=0,005)$ y muerte por cualquier causa $(p=0,002)(69)$. Se recomienda que en los pacientes diabéticos que cursen con un SCA el objetivo del tratamiento debe de contemplar también mantener una glucemia basal menor a $108 \mathrm{mg} / \mathrm{dl}$ y una hemoglobina glicada menor o igual a $6,5 \%$, además de una estrategia terapéutica multifactorial que incluya 
antihipertensivos, antiagregantes, hipolipemiantes y cambios en los estilos de vida (3). Es importante aclarar que no se ha demostrado el beneficio clínico con el tratamiento agresivo con insulina y existe controversia al respecto. Lo que sí se ha establecido de forma experimental es que la insulina reduce la inflamación, coagulación, mejora la función endotelial, la fibrinólisis y reduce la gravedad del infarto en el SCA (70).

\section{CONCLUSIONES}

Los pacientes con hiperglucemia presentan un mayor riesgo de desarrollar un evento coronario, así como de resistencia al tratamiento antiagregante plaquetario. Todo esto se hace evidente al entender la fisiopatología de las vías metabólicas implicadas en la hiperglucemia, las cuales terminan en dos vías comunes: estado proinflamatorio y procoagulante, Io que determina un mayor riesgo coronario $y$ un peor pronóstico. Es fundamental el adecuado control glucémico para impactar en la morbimortalidad, tanto en la prevención primaria como secundaria.

\section{AGRADECIMIENTOS}

Deseamos dejar constancia de nuestro agradecimiento al doctor Juan Manuel Alfaro Velásquez y al doctor Carlos Julio Montoya Guarín por su valiosa colaboración en la revisión de este manuscrito.

Esta revisión fue realizada en el marco de los proyectos de investigación CT447-2013 y 448B-08/15-65, financiados por el Departamento Administrativo de Ciencia, Tecnología e Innovación Colciencias y el Centro para el Desarrollo y la Innovación, CIDI-Universidad Pontificia Bolivariana. KJB y CAVH recibieron financiación del programa de becas de formación en investigación de la Universidad Pontificia Bolivariana. LFH y NGL recibieron financiación del programa de becas para formación doctoral de Colciencias (567 de 2012 y 647 de 2014).

\section{CONFLICTOS DE INTERESES}

Ninguno por declarar.

\section{REFERENCIAS BIBLIOGRÁFICAS}

1. Organización Mundial de la Salud. Enfermedades cardiovasculares [Internet]. 2017 [consultado 2018 Ago 6]. Disponible en: http://www.who.int/es/news-room/factsheets/detail/cardiovascular-diseases-(cvds)

2. Gómez Arbeláez D, Sánchez-Vallejo G, Pérez M, Ronald García G, Arguello JF, Peñaherrera E, et al. Hiperglucemia se asocia a mayor número de desenlaces adversos en individuos latinoamericanos con infarto agudo de miocardio. Clín Investig Arterioscler [Internet]. 2016 Jan [consultado 2018 Aug 6];28(1):918. Disponible en: https://WWW.sciencedirect.com/ science/article/pii/S0214916815001291

3. González-Maqueda I. La enfermedad coronaria deI diabético. Diagnóstico, pronóstico y tratamiento. Rev Esp Cardiol SupI [Internet]. 2007 Jan [consultado 2018 Ago 6];7(H):29H-41. Disponible en: https://www.sciencedirect.com/science/article/pii/S1 131358707752718

4. Lugo JJ, Hurtado EF, Calderón LI, Gómez G, Castro P, Estrada G, et al. Resistencia al ácido acetil salicílico y al clopidogrel: una entidad clínica emergente. Rev Colomb Cardiol [Internet]. 2008 [consultado 2018 Ago 6];4:172-83. Disponible en: https://www.ingentaconnect.com/content/doaj/01205633/2008/00000015/ 00000004/art00006

5. Angiolillo DJ, Suryadevara S. Aspirin and clopidogrel: efficacy and resistance in diabetes mellitus. Best Pract Res Clin Endocrinol Metab. 2009 Jun;23(3):37588. DOI 10.1016/j.beem.2008.12.001.

6. Ang L, Palakodeti $\nabla$, Khalid A, Tsimikas S, Idrees Z, Tran P, et al. Elevated plasma fibrinogen and diabetes mellitus are associated with lower inhibition of platelet reactivity with clopidogrel. J Am Coll Cardiol. 2008 Sep;52(13):1052-9. DOI 10.1016/j.jacc.2008.05.054.

7. Samoš M, Šimonová R, Kovár F, Duraj L, Fedorová J, Galajda P, et al. Clopidogrel resistance in diabetic patient with acute myocardial infarction due to stent thrombosis. Am J Emerg Med. 2014 May;32(5):461-5. DOI 10.1016/j.ajem.2014.01.006.

8. Díaz-Flores M, Baiza-Gutman LA, Ibáñez-Hernández MA, Pascoe-Lira D, Guzmán-Greenfel AM, KumateRodríguez J. Aspectos moleculares del daño tisular inducido por la hiperglucemia crónica. Gac Med Mex. 2004 Jul-Aug; 140(4):437-47.

9. Cassiani CA, Cabrera A. Síndromes coronarios agudos: epidemiología $\searrow$ diagnóstico. Salud Uninorte. 2009;25(1):118-34. 
10. Coll Muñoz Y, Valladares Carvajal J, González Rodríguez $C$. Infarto agudo de miocardio. Actualización de Ia Guía de Práctica Clínica. Guías Prácticas Clínicas [Internet]. 2016 [consultado 2018 Ago 6];6(2):170-90. Disponible en: http://scielo.sld.cu/pdf/rf/\$6n2/rf10206.pdf

11. Manzur F, Arrieta C. Estudio sociológico y del conocimiento de los factores de riesgo de las enfermedades cardiovasculares en la Costa Caribe Colombiana (Estudio Caribe). Rev Colomb Cardiol. 2005;12(3):122-8.

12. Vivas Balcones L. Efecto de un tratamiento intensivo con insulina sobre la reactividad plaquetaria en pacientes con hiperglucemia que ingresan con un síndrome coronario agudo [tesis doctoral]. Madrid: Universidad Complutense; 2010 [consultado 2018 Ago 6]. Disponible en: https://eprints.ucm.es/1 1642/1/T32330.pdf

13. Organización Mundial de la Salud. Informe sobre la situación mundial de las enfermedades no transmisibles [Internet]. 2014 [consultado 2018 Aug 6]. Disponible en: http://apps.who.int/iris/bitstream/handle/10665/149296/ WHO_NMH_NVI_15.1_spa.pdf;jsessionid $=52682 \mathrm{~A} 832 \mathrm{D}$

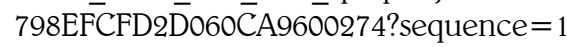

14. Organización Mundial de la Salud. ENT Perfiles de países [Internet]. 2014. Disponible en: http://www. who.int/nmh/countries/col_es.pdf

15. Instituto Nacional de Salud. Observatorio nacional de salud, segundo informe ONS: Mortalidad 19982011 y situación en los municipios de frontera terrestre en Colombia [Internet]. 2013 [cited 2018 Aug 6]. Available from:

https://www.minsalud.gov.co/sites/rid/Lists/BibliotecaDigital/RIDE/IA/INS/Segundo\%20informe\%20ONS.pdf

16. Farmakis D, Parissis J, Lekakis J, Filippatos G. Acute heart failure: Epidemiology, risk factors, and prevention. Rev Esp Cardiol (Engl Ed). 2015 Mar;68(3):245-8. DOI 10.1016/j.rec.2014.11.004.

17. Federación Internacional de Diabetes. Diabetes Atlas [Internet]. 2017 [consultado 2018 Ago 6]. Disponible en: http://diabetesatlas.org/resources/2017-atlas.html

18. Kasper D, Fauci A, Hauser S, Longo D, Jameson JL, Loscalzo J. Harrison. Principios de Medicina Interna. $19^{\text {th }}$ ed. New York: McGraw-Hill; 2012.

19. Fuster $\nabla$, O'Rourke RA, WaIsh RA, Poole-Wilson P. Hurst's the Heart. 12 $2^{\text {th }}$ ed. McGraw-Hill Medical; 2007.

20. Timmis A. Acute coronary syndromes. BMJ. 2015 Oct;351:h5153. DOI 10.1136/bmj.h5153. Erratum in: BMJ. 2015;351:h5849.
21. Rozman C. Farreras Rozman Medicina interna. $17^{\text {th }}$ ed. Barcelona: Elsevier; 2012.

22. Montenegro Cantillo A, Camilo Giraldo G, Castaño JJ. Hiperglucemia: Un marcador independiente de mortalidad y morbilidad en pacientes críticamente enfermos con o sin historia de diabetes, hospitalizados en la clínica Versalles (Manizales, Colombia) 2010-11. Arch Med (Col) [Internet]. 2012 [consultado 2018 Ago 6];12(2):178-84. Disponible en: http://www.redalyc. org/pdf/2738/273825390003.pdf

23. Mansour AA, Wanoose HL. Acute Phase Hyperglycemia among Patients Hospitalized with Acute Coronary Syndrome: Prevalence and Prognostic Significance. Oman Med J. 2011 Mar;26(2):85-90. DOI 10.5001/omj.2011.22.

24. Cherneva ZV, Denchev SV, Gospodinova MV, Milcheva NP, Petrova MG, Cherneva RV. Clinical significance of hyperglycaemia in acute coronary syndrome patients. Acute Card Care. 2011 Dec;13(4):211-8. DOI 10.3109/17482941.2011.629661.

25. Modenesi Rde F, Pena FM, Faria CA, Carvalho RV, Souza NR, Soares Jda S, et al. Influence on prognosis and prevalence of stress hyperglycemia in a cohort of patients with acute coronary syndrome. Rev Bras Ter Intensiva. 2012 Dec;24(4):352-6.

26. Liu XJ, Wan ZF, Zhao N, Zhang YP, Mi L, Wang XH, et al. Adjustment of the GRACE score by HemoglobinA1c enables a more accurate prediction of long-term major adverse cardiac events in acute coronary syndrome without diabetes undergoing percutaneous coronary intervention. Cardiovasc Diabetol. 2015 Aug; 14:110. DOI 10.1186/s12933-015-0274-4.

27. Corrales-Santander H, Manzur-Jattin F, Pacheco-Ayos C, Ardila-Saenz A, Pájaro N Aparicio-Marenco D. Enfermedad coronaria en el paciente con Diabetes Mellitus Tipo 2. Arch Med. 2018 Aug; 14(2):7. DOI 10.3823/1389.

28. Liu HH, Cao YX, Li S, Guo YL, Zhu CG, Wu NQ, et al. Impacts of Prediabetes Mellitus Alone or Plus Hypertension on the Coronary Severity and Cardiovascular Outcomes. Hypertension. 2018 Jun;71(6):1039-1046. DOI 10.1161/HYPERTENSIONAHA.118.11063.

29. Dungan KM, Braithwaite SS, Preiser JC. Stress hyperglycaemia. Lancet. 2009 May;373(9677):1798-807. DOI 10.1016/S0140-6736(09)60553-5.

30. American Diabetes Association. Standards of Medical Care in Diabetes - 2018. J Clin Appl Res Educ [Internet]. 2017 [cited 2018 Aug 6];41(Suppl 1):S3-159. 
Available from: https://diabetesed.net/wp-content/ uploads/2017/12/2018-ADA-Standards-of-Care.pdf

31. Kosiborod M, Rathore SS, Inzucchi SE, Masoudi FA, Wang Y, Havranek EP, et al. Admission glucose and mortality in elderly patients hospitalized with acute myocardial infarction: implications for patients with and without recognized diabetes. Circulation. 2005 Jun; 111 (23):3078-86.

32. Coutinho M, Gerstein HC, Wang Y, Yusuf S. The relationship between glucose and incident cardiovascular events. A metaregression analysis of published data from 20 studies of 95,783 individuals followed for 12.4 years. Diabetes Care. 1999 Feb;22(2):233-40.

33. García Cubero MC, Rodríguez Morales D, Burgoa Alcalaya B. Resistencia al ácido acetilsalicílico y sus repercusiones clínicas. Semer Med Fam. 2008 May;34(5):244-8. DOI 10.1016/S1138-3593(08)71890-2.

34. Marik PE, Bellomo R. Stress hyperglycemia: an essential survival response! Crit Care. 2013 Mar;17(2):305. DOI $10.1186 / \mathrm{cc} 12514$.

35. Acosta Altamirano G, Frías de León MG, Reyes-Montes M del R, Vargas Hernández V, Suárez Cuenca JA. Radicales libres y mecanismos de daño tisular en la diabetes mellitus. Rev Fac Med (Méx) [Internet]. 2011 [consultado 2018 Ago 6];54(3):46-53. Disponible en: http:// www.scielo.org.mx/pdf/facmed/v54n3/v54n3a6.pdf

36. CaIderón Salinas JV, Muñoz Reyes EG, Quintanar Escorza MA. Estrés oxidativo y diabetes mellitus. Rev Educ Bioquímica [Internet]. 2013 Jun [consultado 2018 Aug 6];32(2):53-66. Disponible en: http://www medigraphic. com/cgi-bin/new/resumenI.cgi?IDARTICULO $=74327$

37. Rosado-Pérez J, Mendoza-Núñez VM. Mini-revisión: Inflamación crónica y estrés oxidativo en la diabetes mellitus. Bioquimia [Internet]. 2007 Abr-Jun [consultado 2018 Ago 6];32(2):58-69. Disponible en: http://www.medigraphic.com/cogi-bin/new/resumen. cogi?IDARTICULO $=12738$

38. Cruz Hernández J, Licea Puig ME, Hernández García P. Abraham Marcel EA, Yanes Quesada M. Aldosa reductasa y proteína quinasa $C$ en las complicaciones crónicas de la diabetes mellitus. Rev Mex Patol Clin [Internet]. 2011 [consultado 2018 Ago 6];58(2):102-7. Disponible en: http://www.medigraphic.com/coi-bin/ new/resumen. cogi?IDARTICULO $=29439$

39. Geraldes P, King GL. Activation of protein kinase C isoforms and its impact on diabetic complications.
Circ Res. 2010 Apr;106(8):1319-31. DOI 10.1161/CIRCRESAHA.110.217117.

40. Luna-Ortiz P, Flores-Chávez P, Guarner-Lans V, Machado-Díaz AM, Olivares-Sanromán ME, Martínez-Rosas M. La memoria metabólica y las complicaciones cardiovasculares en el paciente diabético. Rev Mex Anest [Internet]. 2015 Oct [consultado 2018 Ago 6];38(4):24963. Disponible en: http://www.medigraphic.com/coi$\mathrm{bin} /$ new/resumen. coi? IDARTICULO $=62432$

41. Triana Mantilla ME. La hiperolicemia y sus efectos tóxicos. Un concepto patogénico para la micro y macroangiopatía diabética. Rev Cubana Angiol y Cir Vasc [Internet]. 2001 [consultado 2018 Ago 6];2(2):131-41. Disponible en: http://brs.sld.cu/revistas/ang/vol2_2_01/ang10201.pdf

42. López Bojorquez LN. La regulación del factor de transcripción NF-kB. Un mediador molecular en el proceso inflamatorio. Rev Invest Clín [Internet]. 2004 [consultado 2018 Ago 6];56(1):83-92. Disponible en: http://www.scielo.org.mx/scielo.php?script=sci_artte $\mathrm{xt} \& \mathrm{pid}=\mathrm{S} 0034-83762004000100012$

43. Huang TT, Wuerzberger-Davis SM, Wu ZH, Miyamoto S. Sequential modification of NEMO/IKKgamma by SUMO-1 and ubiquitin mediates NF-kappaB activation by genotoxic stress. Cell. 2003 Nov; 115(5):565-76.

44. Galindo-García G, Galván-Plata ME, Nellen-HummeI H, Almeida-Gutiérrez E. Asociación entre hiperolucemia de estrés y complicaciones intrahospitalarias. Rev Med Inst Mex Seguro Soc [Internet]. 2015 [consultado 2018 Ago 6];53(1):6-12. Disponible en: http://www.medioraphic.com/cogi-bin/new/resumen. cgi?IDARTICULO=55094

45. Escárcega RO. El factor de transcripción nuclear kappa en las enfermedades humanas. Rev Med Inst Mex Seguro Soc [Internet]. 2010 [consultado 2018 Ago 6];48(1):55-60. Disponible en: http:// www.medigraphic.com/cgi-bin/new/resumen. coi? IDARTICULO $=36871$

46. Mijajlovic MD, Shulga O, Bloch S, Covickovic-Sternic $\mathrm{N}$, Aleksic $\mathrm{V}$, Bornstein NM. Clinical consequences of aspirin and clopidogrel resistance: an overview. Acta Neurol Scand. 2013 Oct;128(4):213-9. DOI 10.1111/ ane.12111.

47. Abbas Rizvi SK, Mohsin S, Saeed T, Ahmad S, Hussain S. Frequency of Clopidogrel Resistance in Patients of Ischemic Heart Disease. ISCHEMIC Hear Dis. 2013;2:176-80. DOI 10.5083/ejcm.20424884.93. 
48. Marczewski MM, Postula M, Dariusz K. Novel antiplatelet agents in the prevention of cardiovascular complications-focus on ticagrelor. Vasc Health Risk Manag [Internet]. 2010 [cited 2018 Aug 6];6:419-29. Available from: https://www.researchgate.net/publication/44665286

49. Papathanasiou A, Gouderenos J, Tselepis AD. Resistance to aspirin and clopidogrel: possible mechanisms, laboratory investigation, and clinical significance. Hellenic J Cardiol. 2007 Nor-Dec;48(6):352-63.

50. Combescure C, Fontana P, Mallouk N, Berdague P, Labruyere $\mathrm{C}$, Barazer I, et al. Clinical implications of clopidogrel non-response in cardiovascular patients: a systematic review and meta-analysis. J Thromb Haemost. 2010 May;8(5):923-33. DOI 10.1111/j.1538-7836.2010.03809.x.

51. Guevara Arismendy NM, Escobar Gallo GE, Campuzano Maya G. Utilidad clínica de la agregometría plaquetaria. Medicina \& Laboratorio. 2012;18(7-8):311-32.

52. Gorelick PB, Farooq MU. Advances in our understanding of "resistance" to antiplatelet agents for prevention of ischemic stroke. Stroke Res Treat. 2013;2013:727-842. DOI 10.1155/2013/727842.

53. Goodman T, Ferro A, Sharma P. Pharmacogenetics of aspirin resistance: a comprehensive systematic review. Br J Clin Pharmacol. 2008 Aug;66(2):222-32. DOI 10.1111/j.1365-2125.2008.03183.x.

54. Silva F, Rueda-Clausen C, Ardila Y. Resistencia al ácido acetil salicílico. Un reto en el abordaje terapéutico del paciente con alto riesgo cardiocerebrovascular. Acta Med Colomb [Internet]. 2005 Oct-Dic [consultado 2018 Ago 6];30(4):274-80. Disponible en: http:// wWw.redalyc.org/html/1631/163113820005/

55. Papp J, Kenyeres P, Toth K. Clinical importance of antiplatelet drugs in cardiovascular diseases. Clin Hemorheol Microcirc. 2013;53(1-2):81-96. DOI 10.3233/ CH-2012-1578.

56. Qureshi Z, Hobson AR. Clopidogrel "resistance": where are we now? Cardiovasc Ther. 2013 Feb;31(1):3-11. DOI 10.1111/j.1755-5922.2011.00296.x.

57. Gilard M, Arnaud B, Cornily JC, Le Gal G, Lacut K, Le Calvez G, et al. Influence of omeprazole on the antiplatelet action of clopidogrel associated with aspirin: the randomized, double-blind OCLA (Omeprazole CLopidogrel Aspirin) study. J Am Coll Cardiol. 2008 Jan;51(3):256-60. DOI 10.1016/j.jacc.2007.06.064.

58. Angiolillo DJ, Fernandez-Ortiz A, Bernardo E, Ramírez C, Sabaté M, Jimenez-Quevedo p, et al.
Platelet function profiles in patients with type 2 diabetes and coronary artery disease on combined aspirin and clopidogrel treatment. Diabetes. 2005 Aug;54(8):2430-5.

59. Timur AA, Murugesan G, Zhang L, Aung Pp, Barnard J, Wang QK, et al. P2RY1 and P2RY12 polymorphisms and on-aspirin platelet reactivity in patients with coronary artery disease. Int J Lab Hematol. 2012 Oct;34(5):473-83. DOI 10.1111/j.1751553X.2012.01420.X.

60. Gorog DA, Fuster $\nabla$. Platelet function tests in clinical cardiology: unfulfilled expectations. J Am Coll Cardiol. 2013 May;61(21):2115-29. DOI 10.1016/j. jacc.2012.11.080.

61. Matadamas-Zárate C, Hernández-Jerónimo J, PérezCampos E, Majluf-Cruz A. Alteraciones plaquetarias en la diabetes mellitus tipo 2. Arch Cardiol Mex [Internet]. 2009 Dic [consultado 2018 Ago 6]; 79(Supl 2):102-8. Disponible en: http://www pdf/acm/ 7 79s2/ 7 79s2a19.pdf

62. Balasubramaniam K, Viswanathan GN, Marshall SM, Zaman AG. Increased atherothrombotic burden in patients with diabetes mellitus and acute coronary syndrome: a review of antiplatelet therapy. Cardiol Res Pract. 2012;2012:909154. DOI 10.1155/2012/909154.

63. Tang WH, Stitham J, Gleim S, Di Febbo C, Porreca E, Fava $C$, et al. Glucose and collagen regulate human platelet activity through aldose reductase induction of thromboxane. J Clin Invest. 2011 Nov;121(11):446276. DOI 10.1172/JCI59291.

64. Paneni F, Beckman JA, Creager MA, Cosentino F. Diabetes and vascular disease: pathophysiology, clinical consequences, and medical therapy: part I. Eur Heart J. 2013 Aug;34(31):2436-43. DOI 10.1093/eurheartj/eht149.

65. Dunn EJ, Ariëns RA, Grant PJ. The influence of type 2 diabetes on fibrin structure and function. Diabetologia [Internet]. 2005 Jun [cited 2018 Aug 6];48(6):1198206. Available from: http://link.springer.com/10.1007/ s00125-005-1742-2

66. Wang N, Tall AR. Cholesterol in platelet biogenesis and activation. Blood. 2016 Apr 21;127(16):1949-53. DOI 10.1182/blood-2016-01-631259.

67. Kim JD, Park CY, Ahn KJ, Cho JH, Choi KM, Kang JG, et al. Non-HDL cholesterol is an independent risk factor for aspirin resistance in obese patients with type 2 diabetes. Atherosclerosis. 2014 May;234(1):146-51. DOI 10.1016/j.atherosclerosis.2014.01.015. 
68. Siniarski A, Grzybczak R, Rostoff P, Zalewski J, Czubek U, Nessler J, et al. Association of serum levels of lipoprotein A-I and lipoprotein A-I/A-II with high on-treatment platelet reactivity in patients with ST-segment elevation myocardial infarction. Anatol J Cardiol. 2018 Jun; 19(6):37481. DOI 10.14744/AnatolJCardiol.2018.63549.

69. Soldatos G, Cooper ME. Does intensive glycemic control for type 2 diabetes mellitus have long-term benefits for cardiovascular disease risk? Nat Clin Pract Endocrinol Metab. 2009 Mar;5(3):138-9. DOI 10.1038/ncpendmet 1076 .

70. Angeli F, Reboldi G, Poltronieri C, Lazzari L, Sordi M, Garofoli $M$, et al. Hyperolycemia in acute coronary syndromes: from mechanisms to prognostic implications. Ther Adv Cardiovasc Dis. 2015 Dec;9(6):412-24. DOI $10.1177 / 1753944715594528$. 